\title{
Study on the Influence Mechanism of Safety Culture on Safety Accidents in Construction Enterprises Based on Multi-Level Grey Relational Analysis
}

\author{
Tao Feng, Chuanjia Du, Chengjun Wang \\ Xi'an University of Architecture and Technology School of Management, Xi'an 710055, China

\section{基于多层次灰色关联分析的建筑施工企业安全 文化对安全事故影响机理研究} \\ 冯涛, 杜传甲, 王成军 \\ 西安建筑科技大学管理学院, 西安 710055 , 中国
}

\begin{abstract}
Taking the construction enterprises $\mathrm{A}$ and $\mathrm{B}$ as an example, the evaluation index of safety culture grade is established, and the grey relational analysis method is used to select and evaluate the indexes. On this basis, the related data of safety culture and safety accidents are processed with SPSS, and the influence mechanism of safety culture level on safety accidents is further studied. The results show that the higher level of security culture has a more positive impact on the overall situation of safety accidents, the rate of safety accidents, the large amount of accidents and the economic losses of safety accidents. The use of Xi'an 9. 10 to lift the scaffolding demolition accident further demonstrates the results of the empirical analysis.
\end{abstract}

Keywords: Grey relational analysis; safety culture; safety accident; influence mechanism

摘要

以建筑施工企业 $\mathrm{A}$ 和 $\mathrm{B}$ 为例, 建立安全
文化等级评价指标，运用灰色关联分析法对 指标进行笁选、评价, 并在此基础上, 用 SPSS 对安全文化与安全事故的相关数据进行处 理, 进一步安全文化等级对安全事故的影响 机理进行研究。结果显示, 等级越高的安全 文化, 对安全事故整体情况、安全事故率、 较大事故量以及安全事故经济损失有越积 极的影响作用。并利用西安 9-10 提升脚手 架拆除事故对实证分析结果进一步论证。

关键词: 灰色关联分析; 安全文化; 安全事 故; 影响机理

1 引言

安全生产是社会文明进步的标志, 是推 动经济转型升级的重要推动力量 [1]。然而 安全生产事故一直以来都是各个国家所面 临的严峻挑战 [2]。根据对国家安全生产监 督管理总局公布的安全生产简况进行统计 可以发现我国安全生产事故面临的挑战依 然严峻。在对安全事故的分析过程中发现, 主要是由人的不安全行为产生 [3], 而人的 不安全行为被界定为较差安全文化造成的 直接结果 $[4]$ 。

安全文化建设一直是国内外学者持续 关注的焦点问题。Yang Miang Goh, Helen 
Brown 等人指出调查重大事故时安全文化贫 乏为重要因素之一 [5], 王君玲通过问卷调 查的方法, 证实了安全文化各维度对安全绩 效有显著正向影响, 降低建筑企业安全事故 发生率 [6]; 马跃、傅贵证实安全文化 6 个 因子与企业百万吨死亡率存在显著负相关 关系 [7], 杨世军等人提出了安全文化内涵 因素的二维模型, 并进一步归纳出它对安全 绩效的重大作用 [8]。马杰等人在对安全文 化与安全绩效相关性研究的过程中, 引入安 全意识和安全动机作为中介变量, 揭示了他 们之间的作用机理和反作用机理, 建立作用 模型, 对它们之间的关系进行了深层次的挖 掘 $[9]$ 。

从已有的相关文献来看, 现有的实证分 析侧重于安全文化与安全绩效的实证研究, 较少具体到安全事故层面进行分析研究。因 此, 本文以建筑施工企业为样本, 选用 《AQT9005-2008 企业安全文化建设评价准 则》中的指标体系和等级划分标准, 运用多 层次灰色关联评价对建筑施工企业安全文 化建设现状评价, 并按照等级划分标准对其 进行分级, 结合安全管理理论和事故致因理 论来提出假设, 理论联系实际, 定性与定量 相结合, 通过数理统计分析去进行验证分析。

\section{2 建筑施工企业安全文化等级评价}

\section{1 建筑施工企业安全文化等级评价模型 建立}

由于建筑施工企业安全文化系统的因 素较多, 若采用一级关联分析, 因素权重相 对都较小, 为了减小误差, 可采用多级关联 分析, 本文主要讨论二级关联分析。灰色关 联分析评价模型建立的步骤如下:

1) 根据建筑施工企业安全文化指标数 据建立比较序列, 并构建以二者的最优值为 参考值的参考序列。

假设建筑施工企业安全文化评价系统 有 $m$ 个数据序列, 一比较序列有 $n$ 个指标, 构成比较序列, 记为 $X_{i}(j)$ 。

$$
\begin{aligned}
& X_{i}(j)=\left[x_{i}(1), x_{i}(2), \cdots \cdots, x_{i}(n)\right] \\
& j=1,2, \cdots \cdots, n
\end{aligned}
$$

设 $X_{0}(j)$ 为参考序列, 参考序列为各个 指标的最优值。
$X_{i}(j)$ 为比较序列, 即为建筑施工企业 安全文化评价指标的实际数值。

则 $X_{i}(j)$ 与 $X_{0}(j)$ 序列的绝对差:

$$
\Delta_{o i}(j)=\left|X_{0}(j)-X_{i}(j)\right|
$$

2) 求参考数列与比较数列的灰色关联 系数。

在 2) 的基础上, 计算关联系数, 比较 序列 $X_{i}$ 对于参考序列 $X_{0}$ 的关联系数为:

$$
L_{o i}(j)=(\Delta \min +\rho \Delta \max ) /\left(\Delta_{o i} k+\rho \Delta \max \right)
$$

其中, $\Delta \min =\min _{i} \Delta_{o i}(j) ; \Delta \max =\max _{i} \Delta_{o i}(j)$; $\rho$ 为分辨系数。

分辨系数的存在是为了减小 $\Delta \max$ 数值 过大而失真的影响, 提高关联系数之间的差 异显著性 [10], 取值区间为 $(0,1)$, 一般情 况下 $\rho=0.5$ 为宜。

3) 求关联度并对其进行排序。

如果两个比较序列与参考序列的形状 在各个时刻都相像, 那么关联系数则可以记 为 1 , 关联度一样为 1 。时间序列不可能相 交, 关联度大于 0 。所以, 比较序列关联度 可用与参考序列的关联度和时间序列关联 度的最优值表示, 即为

$$
r_{o i}=\frac{1}{n} \sum_{j=1}^{n} L_{o i},
$$

其中 $r_{o i}$ 为 $X_{i}(j)$ 序列与 $X_{0}(j)$ 序列的关联度。 关联度主要是比较各个 $X_{i}(t)$ 与 $X_{0}(t)$ 谁大谁 小, 关联度越大, 说明关系更紧密。这样也 就可以看出建筑施工企业安全文化指标有 无达到要求。

4) 建筑施工企业安全文化评价指标体 系指标过多, 关联系数也相应有很多, 用传 统的灰色关联评价计算繁琐, 难免会出现纰 漏, 分辨系数的取值大小, 客观上反映了系 统因素对关联度的间接影响程度, 综合考量, 对重要程度不同的各因素赋权, 则加权灰色 关联度为:

$$
r_{o i}=\sum_{j=1}^{n} L_{o i}(j) \bullet w_{i}(j), i=1,2, \cdots \cdots, m
$$

其中, $w_{i}(j)$ 是数列中各项对应的指标权重 
且满足: $w_{i}(j) \geq 0, \sum_{j=1}^{n} w_{i}(j)=1$ 。

\section{2 建筑施工企业安全文化评价模型应用}

本文在明确了指标权重的前提下, 运用 多层次灰色关联分析对安全文化建设情况 进行科学评价。具体步骤如下:

数据搜集。本文选取西安市 $\mathrm{A} 、 \mathrm{~B}$ 两家
企业作为样本, 进行调研, 了解两家施工企 业安全化建设的现状。本调查对决策层、管 理层和员工层人员分别发放问卷, 其中决策 层和管理层 60 份, 工人层 141 份, 回收率 均为 $93 \%$, 将回收的数据用 SPSS 处理, 得 到 A、B 企业的安全文化相关数据, 逐级进 行关联度的计算, 可得建筑施工企业安全文 化关联度计算结果, 如表 1 所示。

表 1 建筑施工企业安全文化关联度计算结果

\begin{tabular}{cccc}
\hline 等级 & $\mathrm{r}_{01}$ & $\mathrm{r}_{02}$ & \multicolumn{1}{c}{ 备注 } \\
\hline & & & 第一层级: 本能反应阶段 $(0.35$ 以下 $)$ \\
安全文化 & 0.81 & 0.74 & $\begin{array}{r}\text { 第二层级: 被动管理阶段 }(0.35-0.49) \\
\text { 关联度 }\end{array}$ \\
& & 第三层级: 主动管理阶段 $(0.50-0.64)$ \\
& & 第四层级: 员工参与阶段 $(0.65-0.79)$ \\
安全文化 & 团队互助阶段 & 员工参与阶段 & 第五层级: 团结互助阶段 $(0.80-0.90)$ \\
等级判定 & & 第六层级: 持续改进阶段 $(0.90$ 以上
\end{tabular}

通过对 A、B 两家企业的评价, 我们可以 看出 $\mathrm{A}$ 企业处于团结互助阶段, B 企业在员 工参与阶段。

3 建筑施工企业安全文化对安全事故影响 机理研究

3.1 建筑施工企业安全文化对安全事故影 响机理研究模型的构建

\subsection{1 研究假设的提出及模型的构建}

基于安全管理理论和事故致因理论, 在相 关文献研究的基础上提出如下假设:

$H 1$ ：建筑施工企业安全文化等级越高, 安 全行为越多。

H2: 建筑施工企业安全文化等级越高, 物 态就越安全。

H3: 建筑施工企业安全行为越多, 物态就 越安全。

H4: 安全行为对安全事故有直接的显著的 负向影响。

H5: 安全物态对安全事故有直接的显著的 负向影响。

以安全行为和安全物态作为中间变量, 建 立图 1 所示的研究模型。

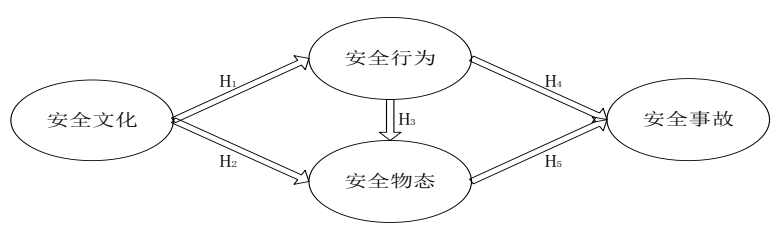

图 1. 建筑施工企业安全文化对安全事故影响机理模型

\section{1.2 研究变量的界定和测量}

通过对文献的分析, 本研究安全行为和安 全物态量表借鉴杨世军（2012）的测量题项, 采用里克特七点量表, 安全事故量表包括安全 事故整体情况、安全事故率低、较大事故量少、 安全事故损失少等条目。由完全不符合到完全
符合分别给予 1 分到 7 分, 分数越高代表公 司的安全绩效越好。安全文化等级则由前文评 价体系评价所得。

\section{2 研究数据搜集与检验}

\subsection{1 调研方法}

为检验本研究模型的合理性, 我们选取了 
陕西的 2 个典型的建筑施工企业, 从中邀请了 安全生产相关人员,通过调查对其 2014-2016 年的安全生产数据进行收集。本次调研通过例 会安排参加调研的人们填写调查问卷, 问卷填 好以后当场回收。

\section{2 .2 样本分析}

本次调研发出 210 份问卷, 回收 201 份, 回收率为 $95.7 \%$ 。由于本次调研为现场调研, 问题当场解释, 问卷当场检查, 因此 201 份问 卷无遗漏项。

\section{2 .3 信度和效度检验}

(1) 信度分析

本文通过 SPSS20.0 中的 Cronbach' $\mathrm{s}$ 系 数对潜变量和整体量表的组合信度验证, 结果 Cranach’ s a>0.7, 因此本文调查问卷有很 好的信度, 数据可靠。

(2) 效度分析

本研究所采用的量表是基于前人的量表, 并且经过高校老师和企业安全人员的论证, 故 有较好的内容效度。而对结构效度的评价, 本 文对有效样本进行 KMO 抽样适当性检验和 Bartlett 球形检验, 经检验 KMO 值是 0.933,
远大于 0.5 ; Sig. 为 0.000 , 小于 0.05 , Bartlett 检验显著, 因此本文问卷通过效度 检验, 可以进行因子分析。

\section{3 统计分析和结果讨论}

描述性分析结果 (篇幅有限, 未附) 说明 问卷题目有较高辨识度, 并通过 SPSS 中主成 分分析法对测量指标进行因子提取, 表明安全 文化、安全行为、安全物态和安全事故可由相 关题目进行体现。

\section{3.1 相关性分析}

通过 SPSS20.0 中的相关分析, 得到表 2 中各变量间的相关系数。安全文化与安全行为 和安全物态均显著正相关, 安全行为对安全物 态正相关, 安全行为与安全物态对安全事故正 相关。相关分析结果可以初步验证了前文的理 论假设模型。

\subsection{2 回归分析}

本文建立多个回归分析模型, 对建筑施工 企业安全文化建设水平于安全事故的关系假 设进一步验证, 通过 SPSS20.0 软件分析工具 对数据处理, 得到表 3 的回归分析结果。

\begin{tabular}{lllllllll}
\multicolumn{8}{c}{ 表 2. 控制变量描述性统计及相关系数 } \\
\hline 变量 & 平均值 & 方差 & 学历 & \multicolumn{1}{l}{ 从业时长 } & 安全文化 & 安全行为 & 安全物态 & 安全事故 \\
\hline 学历 & 2.66 & 1.097 & 1 & & & & & \\
从业时长 & 2.09 & 0.72 & $0.289 * *$ & 1 & & & & \\
安全文化 & 4.33 & 0.98 & -0.042 & $0.245 * *$ & 1 & & & \\
安全行为 & 3.98 & 0.91 & -0.087 & 0.102 & $0.301 * *$ & 1 & & \\
安全物态 & 4.05 & 0.78 & -0.061 & -0.010 & $0.213 *$ & $0.601 * *$ & 1 & \\
安全事故 & 4.58 & 0.81 & -0.103 & 0.126 & $0.294 * *$ & $0.623 * *$ & $0.598 * *$ & 1 \\
\hline 注: 采用 Pearson 相关分析; *表示在 0.05 水平 (双侧) 显著相关; **表示在 0.01 水平 (双侧) 上显 \\
著相关 [11], 表 3-2 同。
\end{tabular}

表 3. 多元分步回归分析结果

\begin{tabular}{|c|c|c|c|c|c|c|c|c|c|c|}
\hline \multirow{2}{*}{$\begin{array}{l}\text { 结果 } \\
\text { 变量 }\end{array}$} & \multicolumn{8}{|c|}{ 安全事故 } & \multirow{2}{*}{$\begin{array}{l}\begin{array}{l}\text { 安全 } \\
\text { 行为 }\end{array} \\
\text { 模型 } 9 \\
\end{array}$} & \multirow{2}{*}{$\begin{array}{l}\text { 安全 } \\
\text { 物态 } \\
\text { 模型 } 10\end{array}$} \\
\hline & 模型 1 & 模型 2 & 模型 3 & 模型 4 & 模型 5 & 模型 6 & 模型 7 & 模型 8 & & \\
\hline 年龄 & -0.066 & -0.069 & 0.029 & 0.020 & -0.018 & -0.024 & -0.031 & -0.154 & 0.094 & 0.050 \\
\hline 学历 & 0.063 & 0.021 & 0.036 & 0.018 & 0.059 & 0.030 & 0.024 & 0.045 & 0.119 & 0.112 \\
\hline $\begin{array}{l}\text { 安全 } \\
\text { 文化 }\end{array}$ & - & $0.328^{* *}$ & - & $0.156^{* *}$ & - & $0.223^{*}$ & - & $0.248^{* *}$ & $0.698^{* *}$ & $0.567^{*}$ \\
\hline $\begin{array}{l}\text { 安全 } \\
\text { 行为 }\end{array}$ & - & - & $0.618^{* *}$ & $0.572 *$ & - & - & $0.555^{* *}$ & $0.449^{* *}$ & & \\
\hline $\begin{array}{l}\text { 安全 } \\
\text { 物态 }\end{array}$ & - & - & - & - & $0.061^{*}$ & $0.506^{* *}$ & $0.469^{*}$ & $0.110^{*}$ & & \\
\hline$\Delta \mathrm{R} 2$ & 0.011 & 0.105 & 0.376 & 0.292 & 0.365 & 0.050 & 0.089 & 0.255 & & \\
\hline
\end{tabular}


本文以表格的方式对结果进行解释讨论, 如表 4, 验证建筑施工企业安全文化与安全事 故之间的关系, 同时对安全文化对安全事故的 影响机理也进行了验证。

同理, 对安全文化与安全事故率、较大事
故量和事故经济损失进行回归分析。本文根据 回归分析的结果, 得到图 2 至图 5 的模拟回归 曲线。

表 4. 多元分步回归分析结果讨论

\begin{tabular}{|c|c|c|c|}
\hline 序号 & 模型表示 & 回归系数 & 结论 \\
\hline 1 & 安全事故对控制变量 & & \\
\hline 2 & 安全事故对控制变量和安全文化 & $0.328(\mathrm{p}<0.01)$ & 选题成立 \\
\hline 3 & 安全事故对控制变量和安全行为 & $0.618(\mathrm{p}<0.01)$ & \\
\hline 4 & 安全事故对控制变量和安全文化、安全行为 & $\begin{array}{l}0.156 \quad(\mathrm{p}<0.01) \\
0.572(\mathrm{p}<0.01)\end{array}$ & \\
\hline 5 & 安全事故对安全物态和控制变量 & $0.061 （ \mathrm{p}<0.01)$ & \\
\hline 6 & 安全事故对控制变量和安全文化、安全物态 & $\begin{array}{l}0.223(\mathrm{p}<0.01) \\
0.506(\mathrm{p}<0.01)\end{array}$ & 假设 5 成立 \\
\hline 7 & 安全事故对控制变量和安全行为、安全物态 & $\begin{array}{l}0.555(\mathrm{p}<0.01) \\
0.469(\mathrm{p}<0.01)\end{array}$ & 假设 3、4、5 成立 \\
\hline 8 & $\begin{array}{l}\text { 安全事故对控制变量和安全文化、安全行为、 } \\
\text { 安全物态 }\end{array}$ & $\begin{array}{l}0.248 \quad(p<0.01) \\
0.449(p<0.01) \\
0.110(p<0.01)\end{array}$ & $\begin{array}{l}\text { 理论模型得到进一步 } \\
\text { 验证 }\end{array}$ \\
\hline 9 & 安全行为对安全文化和控制变量 & $0.698 \quad(\mathrm{p}<0.01)$ & 假设 1 成立 \\
\hline 10 & 安全物态对安全文化和控制变量 & $0.567(\mathrm{p}<0.01)$ & 假设 2 成立 \\
\hline
\end{tabular}

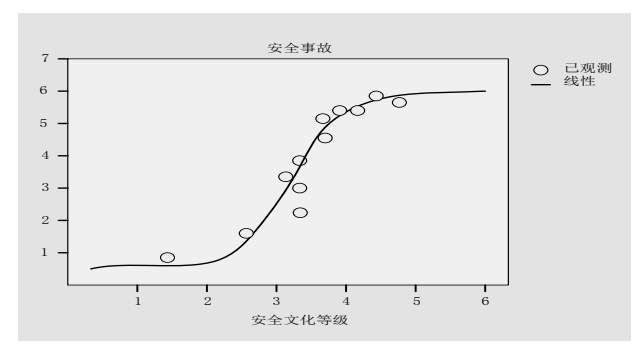

图 2. 安全文化与安全事故整体情况拟合曲线图

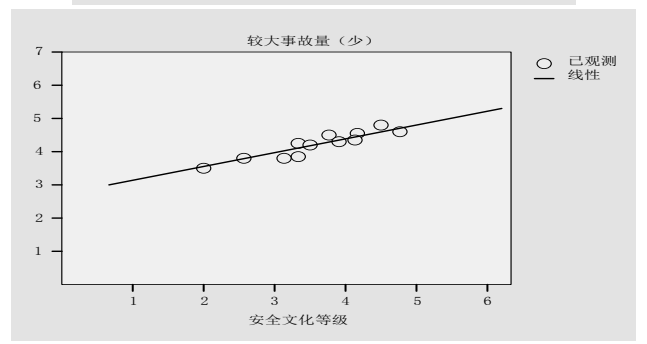

图 3. 安全文化与较大事故量 (少) 拟合曲线图 


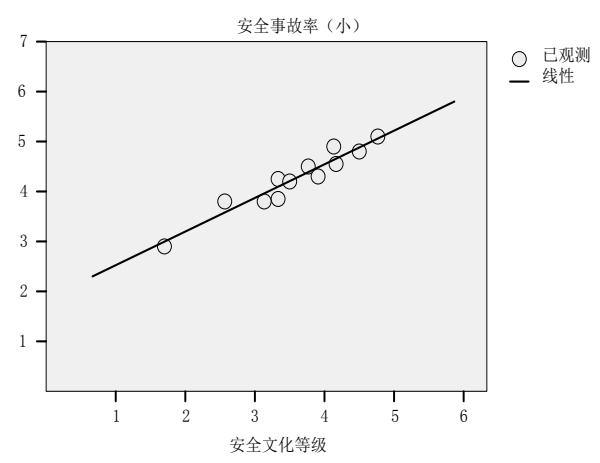

图 4. 安全文化与安全事故率 (小) 拟合曲线图

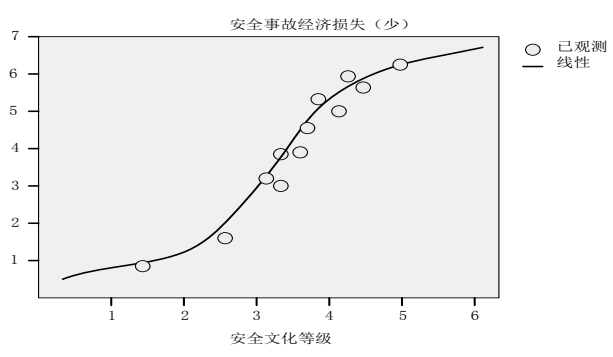

图 5. 安全文化与安全事故损失 (少) 曲线图

\subsection{3 结果讨论}

建筑施工企业安全文化对安全事故的整 体情况有着显著地正向影响。总体来说, 在安 全文化等级为一、二级比较低的时候, 安全文 化对安全事故影响的效果不是很明显, 但是随 着建设水平的不断提升, 对安全事故的影响比 较显著, 但是到了五级之后, 对安全事故的作 用程度趋于平缓, 在这个阶段, 安全事故的发 生与安全文化建设的水平无关, 如果发生, 很 大可能是因为不可抗力。

安全文化建设水平对安全事故率的影响比较 直接, 安全文化等级越高, 安全事故率就越低, 较大事故量也随之减少。在某种程度上安全事 故经济损失与较大事故量有着一定关系, 而由 我们的数据可得安全文化与安全事故经济损 失的关系则如图 5 所示, 在安全文化等级达到 了五级之后, 安全文化建设水平对安全事故经 济损失就会比较平缓, 在这个阶段, 人为因素 的事故是次要矛盾, 不可抗力引起的事故才是 主要矛盾, 这时安全事故一旦发生, 则相对比 较严重, 但是安全文化建设主要是对事故发生
后的后续防范处理影响较大, 因此重点建设安 全事故处理方法, 以预防事故状态进一步扩大 或减少二次事故带来的经济损失。

\section{4 结论}

1）基于灰色关联分析的评价方法, 对建 筑施工企业安全文化等级进行评定, A 企业处 于团结互助等级, B 处于员工参与阶段, 跟事实相符。

2) 基于安全管理理论和事故致因理论, 提出研究假设, 从而进行相关分析和回归分析。 研究结果表明, 安全文化建设水平通过安全事 故和安全物态对安全事故产生影响, 安全文化 等级越高, 安全事故越能往积极的方向发展。

3) 基于实证分析, 研究结论更加客观。 研究结果可以帮助建筑施工企业加强安全文 化建设, 为减少企业安全事故量、降低安全事 故率以及损失的实践提供借鉴。

\section{参考文献}

[1] Yu H. Study on performance evaluation of government comprehensive supervision for safety production based on balanced score 
card --- a case study in Shandong Province, China. Journal of risk analysis and crisis response, 2016, 6 (2): 85-94.

[2] 李永娟,蒋丽, 胥遥山,王璐璐. 工作压力与 社会支持对安全绩效的影响, 心理科学 进展, 2011, (03):318-327.

[3] 张孟春,方东平. 建筑工人不安全行为产 生的认知原因和管理措施. 土木工程学 报,2012, S2:297-305.

[4] 杨月江, 傅贵. 论安全文化理念是预防事故 之根本. 中国矿业,2007, 05:33-35.

[5] Yang M G, Brown H, Spickett J, Applying systems thinking concepts in the analysis of major incidents and safety culture. Safety Science,2010,48:302-309.

[6] 王君玲. 建筑企业安全文化对安全绩效 影响的实证研究. 中国安全生产科学技
术,2014, 08:125-129.

[7] 马跃,傅贵,藏亚丽. 企业安全文化结构及 其与安全业绩关系研究, 中国安全科学 学报,2015,05:145-150.

[8] 杨世军,袁玥,陆绍凯. 建筑行业安全文化 研究. 建筑经济,2012,06:100-103.

[9] 马杰, 李㻈, 张玲玲. 煤矿安全文化与安全 绩效的关系. 辽宁工程技术大学学报(社 会科学版),2009, 03:236-239.

[10] 刘芳,许程洁. 基于灰色系统关联分析的 施工企业安全投资研究. 建筑管理现代 化,2009, (03):209-212.

[11] 曹庆仁, 李凯, 刘丽娜. 煤矿安全文化对员 工行为安全影响作用的实证研究. 中国安 全科学学报,2011, 21(4): 143-149. 